\title{
New proposed spherical slotted antenna covered by the layers of dielectric material and plasma
}

\author{
Osman Shakirovich Dautov ${ }^{1}$, Mohammad Sadon AL-Abadi ${ }^{2}$, Haidar N. Al-Anbagi ${ }^{3}$ \\ ${ }^{1,2}$ Department of Radio-Engineering and Telecommunications Systems, University of KAI, Russia \\ ${ }^{2,3}$ Department of Communications Engineering, University of Diyala, Iraq
}

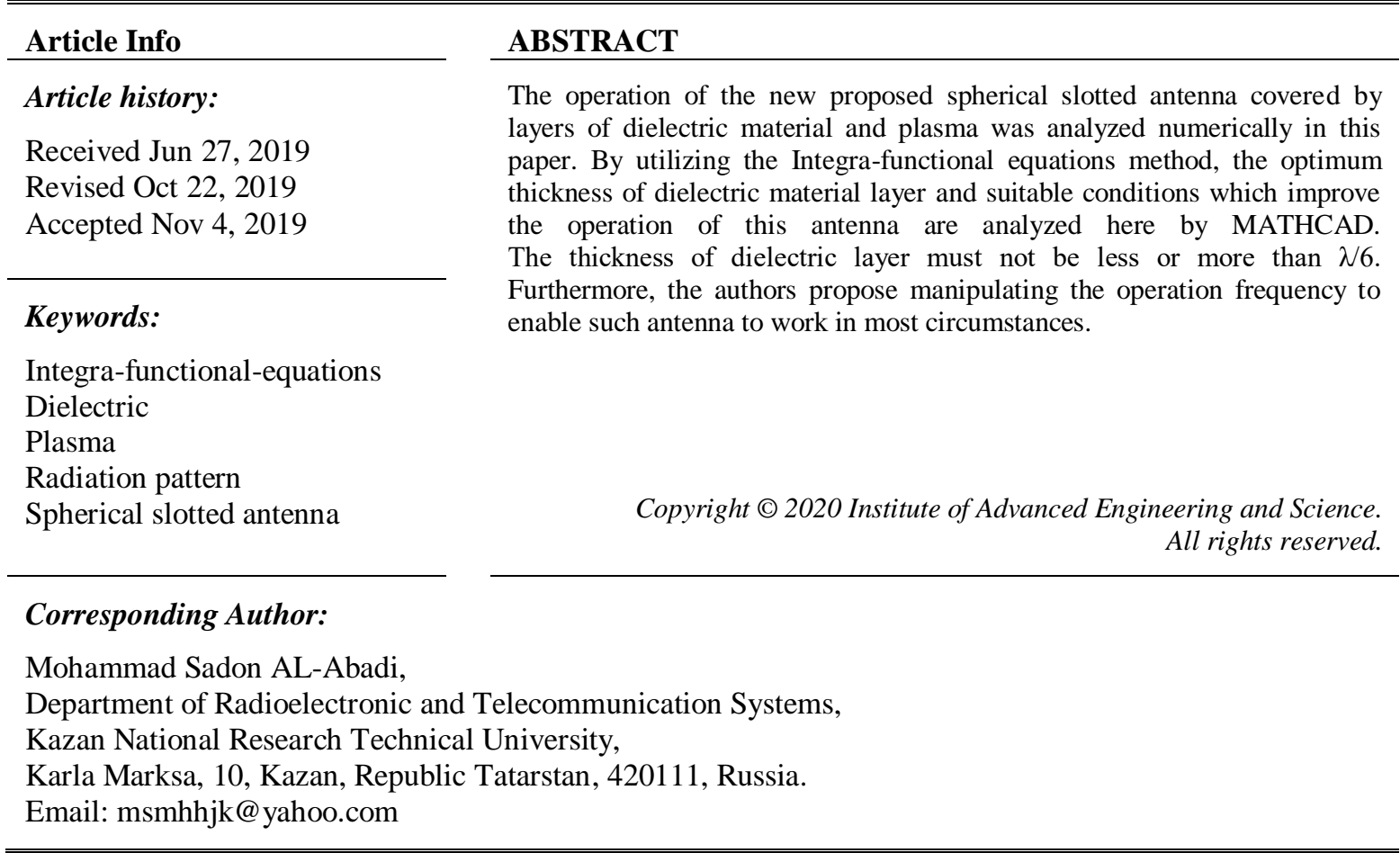

\section{INTRODUCTION}

Antennas with spherical geometries have been investigated by many researchers. These investigations are based on horizontal rectangular and zonal slots on a conducting spherical cavity [1-14]. Optimum thickness of single dielectric coating shifts the peak of the main beam towards smaller value of $\theta$ [15]. The results in [16] where the frequency range is $10 \mathrm{GHz}$, show that there is no significant effect of the change of supplied frequency on the radiation pattern. In addition, increasing the thickness of the coating layer leads to a decrease in the level of radiation and that also can lead to a change in the directivity causing consequently the side lobes to appear.

As it is known, plasma, which is the fourth state of matter, has many applications in electromagnetic engineering [17-22]. Recently it has played an important role in military applications where plasma can transmit, reflect, and absorb the electromagnetic waves under various circumstances. Based on the importance of plasma, there are many studies that investigated the behavior of plasma with respect to slot antenna [23-26]. According to [16], the results clearly show that the maximum radiation pattern and directivity can be obtained when the radius of the spherical slotted antenna covered by plasma layer is $\lambda / 2$ and the optimum thickness for the plasma layer is $\lambda / 15$. Therefore, in this paper, the authors will adopt these results and assume the suitable size of the proposed spherical slotted antenna at radius $\lambda / 2$ and also assume the thickness of plasma layer is $\lambda / 15$. This background nformation directly leads to the main goals of this paper to deal with the problem of antenna dielectric coating thickness and its consequences on the antenna performance in addition to the problem of plasma layer density wich also affects the radiation pattern of the antenna 


\section{PROPOSED METHDOLOGY}

In this study, the radiation pattern of a spherical slotted antenna covered by a dielectric material as a first layer and plasma as a second layer is considered and examined. The geometric surface for spherical slotted antenna system is shown in Figure 1. The dielectric cover parameters are denoted by $\varepsilon_{o}, \mu_{o}$, the plasma parameters by $\varepsilon_{i}, \mu_{i}$, and the outer space parameters by $\varepsilon_{i}, \mu_{i}$. Relying on the results in [16] and [27], we assume in our research the operation frequency is $1 \mathrm{GHz}$ with angular frequency denoted by $\omega$, the radius of sphere $R_{o}$ will be equal to $\lambda / 2$. The dielectric constant of dielectric layer $\left(R_{1}\right)$, which surrounds the spherical slotted antenna, is 2.1 and the second layer is plasma layer with plasma frequency denoted by $\omega_{i}$ whose radius of $\left(R_{2}\right)$.

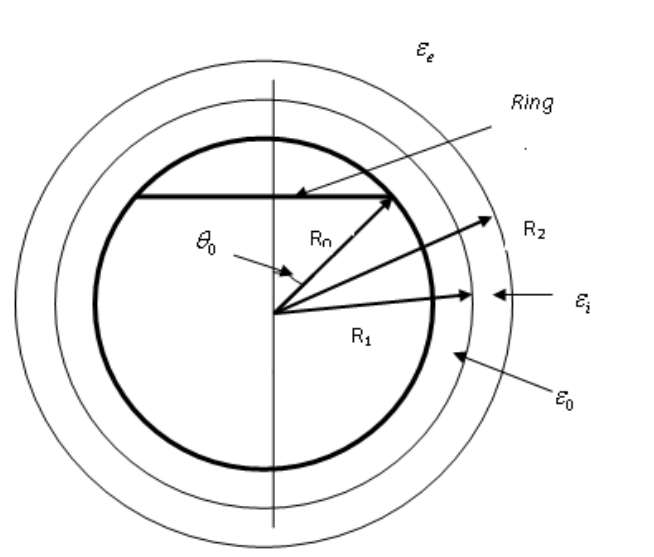

Figure 1. The spherical slotted antenna surrounded by a layer of dielectric material in plasma

In the next sections, the authors will numerically investigate to find out the optimum thickness of dielectric layer that allows the electromagnetic waves to propagate as a maximum radiation pattern to the free space. Additionally, this paper investigates also the possibility of the electromagnetic waves being prevented by the plasma layer and enables it to propagate to the free space. The model which used here is demonstrated with the help of Integra-functional equations (IFE) [28, 29].

\section{ANALYTICAL FORMULATION}

In a homogeneous dielectric body $v_{i}$ with $\varepsilon_{i}$ and $\mu_{i}$ parameters, the Integra-functional equation for an external magnetic field is:

$$
\left.\oint_{S}\left\{\frac{\left(\stackrel{r}{\left.H_{e} v\right)}\right.}{\mu_{r}} \nabla_{s} G_{i}-\left[\stackrel{r}{r} \underset{H_{e}}{r}\right] \nabla_{s} G_{i}\right]-\varepsilon_{r}[\stackrel{r}{r} \underset{r}{r} \stackrel{r}{r}] G_{i}\right\} d s=\stackrel{r}{H_{o i}}\left(\stackrel{r}{r} \in V_{e}\right),
$$

Where:

$\mathrm{S}$ - the surface of the body,

$G_{i}$ - Green's function of a homogeneous space with body parameters,

$\nabla_{s}$ - Gradient operator with respect to coordinates of integration point $\vec{r}_{s}$,

$\vec{H}_{o i}$ - Unperturbed field of external sources located in $V_{i}$,

$\vec{H}_{e}$ - The desired external field consisting of an unperturbedfield of external sources $\vec{H}_{o e}$, located in $V_{c m e} \subset V_{e}$,

The outer region may be non-uniform and, accordingly $\varepsilon_{e}, \mu_{e}$ may be functions of the coordinates.

Usage Integra-functional equation has the form:

$$
L\left(l, H_{e}\right)=\pi \int_{l}\left\{H_{e} \frac{\partial\left(\rho_{s} G_{i 1}\right)}{\partial v}-\frac{\varepsilon_{i}}{\varepsilon_{e}} \frac{\partial\left(\rho_{s} H_{e}\right)}{\partial v} G_{i 1}\right\} d l= \begin{cases}H_{o i} & \left(\stackrel{r}{r} \in V_{e}\right) \\ -H_{p i} & \left(r \in V_{i}\right)\end{cases}
$$


The total field inside the dielectric cover will be presented in the form of two components:

$$
H_{e}=H_{o e}+H_{p e}, \quad\left(r \leq R_{1}\right)
$$

Where:

$$
\begin{aligned}
& H_{o e}=\sum_{n=1}^{\infty} D_{n} h_{n}\left(k_{o} \stackrel{r}{r}\right), \\
& H_{p e}=\sum A_{n} j_{n}\left(k_{o} \stackrel{r}{r}\right), \\
& h_{n}\left(k_{o} \stackrel{r}{r}\right)=\xi_{n}{ }^{(2)}\left(k_{o} r\right) P_{n}{ }^{1}(\cos \theta), j_{n}\left(k_{o} \stackrel{r}{r}\right)=\psi_{n}\left(k_{o} r\right) P_{n}^{1}(\cos \theta) .
\end{aligned}
$$

The functions $\xi_{n}^{(2)}(z), \psi(z)$ are radial spherical functions associated with the cylindrical functions of Hankel and Bessel relations, respectively:

$$
\xi_{n}^{(2)}(z)=\sqrt{\frac{\pi}{2 z}} H_{n+\frac{1}{2}}^{(2)}(z), \quad \psi(z)=\sqrt{\frac{\pi}{2 z}} J_{n+\frac{1}{2}}(z),
$$

$P_{n}^{1}(\cos \theta)$ - associated Legendre functions.

The field in outer space $\left(r \geq R_{2}\right)$ has the following representation:

$$
H_{e}=\sum_{n=1}^{\infty} B_{n} h_{n}\left(k_{e} \stackrel{r}{r}\right)
$$

Here, we use the decomposition of the function $G_{i 1}$ on spherical harmonics to calculate the integral:

$$
G_{i 1}=-\frac{i k_{i}}{2 \pi} \sum_{n=1}^{\infty} \frac{2 n+1}{n(n+1)} \psi_{n}\left(k_{i} r^{\prime}\right) \xi_{n}{ }^{(2)}\left(k_{i} r\right) P_{n}^{1}\left(\cos \theta^{\prime}\right) P_{n}^{1}(\cos \theta)
$$

As we noted in (2), the integration operation is the sum of the integration along the internal and external contours:

$$
L\left(l, H_{e}\right)=L\left(l^{\prime}, H_{e}\right)+L\left(l^{\prime \prime}, H_{e}\right)
$$

Taking in consideration the orthogonality of the associated Legendre polynomials:

$$
\int_{0}^{\pi} P_{n}{ }^{1}(\cos \theta) P_{n^{\prime}}{ }^{1}(\cos \theta) \sin \theta d \theta= \begin{cases}0 & \left(n^{\prime} \neq n\right) \\ \frac{2 n(n+1)}{2 n+1} & \left(n^{\prime}=n\right)\end{cases}
$$

From equations (4), (5), and (6), the values of the integral operators with respect to (8) can be written as follows:

$$
\begin{aligned}
& L\left(l^{\prime}, H_{o e}\right)=\frac{i}{4} \sum_{n=1}^{\infty} D_{n}\left\{\begin{array}{l}
\alpha_{n}^{h}\left(R_{1}\right) h_{n}\left(k_{i} \stackrel{r}{r}\right)\left(r>R_{1}\right) \\
\beta_{n}^{h}\left(R_{1}\right) j_{n}\left(k_{i} r\right)\left(r<R_{1}\right)
\end{array}\right. \\
& \alpha_{n}^{h}\left(R_{1}\right)=k_{i} R_{1}\left\{\xi_{n}^{(2)}\left(k_{0} R_{1}\right)\left[k_{i} R_{1} \psi_{n}\left(k_{i} R_{1}\right)\right]^{\prime}-\frac{\varepsilon_{i}}{\varepsilon_{0}}\left[k_{0} R_{1} \xi_{n}^{(2)}\left(k_{0} R_{1}\right)\right]^{\prime} \psi_{n}\left(k_{i} R_{1}\right)\right\} \\
& \beta_{n}^{h}\left(R_{1}\right)=k_{i} R_{1}\left\{\xi_{n}^{(2)}\left(k_{0} R_{1}\right)\left[k_{i} R_{1} \xi_{n}^{(2)}\left(k_{i} R_{1}\right)\right]^{\prime}-\frac{\varepsilon_{i}}{\varepsilon_{0}}\left[k_{0} R_{1} \xi_{n}^{(2)}\left(k_{0} R_{1}\right)\right]^{\prime} \xi_{n}^{(2)}\left(k_{i} R_{1}\right)\right\} .
\end{aligned}
$$


In the same manner, for the scattering field:

$$
\begin{aligned}
& L\left(l^{\prime}, H_{p e}\right)=\frac{i}{4 \pi} \sum_{n=1}^{\infty} A_{n}\left\{\begin{array}{l}
\alpha_{n}{ }^{j}\left(R_{1}\right) h_{n}\left(k_{i} r\right) \\
\beta_{n}{ }^{j}\left(R_{1}\right) j_{n}\left(k_{i} r\right)
\end{array}\right. \\
& \alpha_{n}^{j}\left(R_{1}\right)=k_{i} R_{1}\left\{\psi_{n}\left(k_{0} R_{1}\right)\left[k_{i} R_{1} \psi_{n}\left(k_{i} R_{1}\right)\right]^{\prime}-\frac{\varepsilon_{i}}{\varepsilon_{0}}\left[k_{0} R_{1} \psi_{n}\left(k_{0} R_{1}\right)\right]^{\prime} \psi_{n}\left(k_{i} R_{1}\right)\right\} \\
& \beta_{n}^{j}\left(R_{1}\right)=k_{i} R_{1}\left\{\psi_{n}\left(k_{0} R_{1}\right)\left[k_{i} R_{1} \xi_{n}^{(2)}\left(k_{i} R_{1}\right)\right]^{\prime}-\frac{\varepsilon_{i}}{\varepsilon_{0}}\left[k_{0} R_{1} \psi_{n}\left(k_{0} R_{1}\right)\right]^{\prime} \xi_{n}^{(2)}\left(k_{i} R_{1}\right)\right\}
\end{aligned}
$$

The integration over the outer contour gives the following:

$$
\begin{aligned}
& L\left(l^{\prime \prime}, H_{e}\right)=-\frac{i}{4} \sum_{n=1}^{\infty} B_{n}\left\{\begin{array}{l}
\alpha_{n}{ }^{h}\left(R_{2}\right) h_{n}\left(k_{i} r\right)\left(r>R_{2}\right) \\
\beta_{n}{ }^{h}\left(R_{2}\right) j_{n}\left(k_{i} r\right)\left(r<R_{2}\right)
\end{array}\right. \\
& \alpha_{n}{ }^{h}\left(R_{2}\right)=k_{i} R_{2}\left\{\xi_{n}^{(2)}\left(k_{e} R_{2}\right)\left[k_{i} R_{2} \psi_{n}\left(k_{i} R_{2}\right)\right]^{\prime}-\frac{\varepsilon_{i}}{\varepsilon_{e}}\left[k_{e} R_{2} \xi_{n}^{(2)}\left(k_{e} R_{2}\right)\right]^{\prime} \psi_{n}\left(k_{i} R_{2}\right)\right\} \\
& \beta_{n}^{h}\left(R_{2}\right)=k_{i} R_{2}\left\{\xi_{n}^{(2)}\left(k_{e} R_{2}\right)\left[k_{i} R_{2} \xi_{n}^{(2)}\left(k_{i} R_{2}\right)\right]^{\prime}-\frac{\varepsilon_{i}}{\varepsilon_{e}}\left[k_{e} R_{2} \xi_{n}^{(2)}\left(k_{e} R_{2}\right)\right]^{\prime} \xi_{n}^{(2)}\left(k_{i} R_{2}\right)\right\}
\end{aligned}
$$

Since there are no external sources inside the plasma layer, $H_{0 i}$ is equal to zero, therefore, substituting (10), (13), and (16) into (2), we obtain for $r>R_{2}$ :

$$
D_{n} \alpha_{n}^{h}\left(R_{1}\right)+A_{n} \alpha_{n}^{j}\left(R_{1}\right)-B_{n} \alpha_{n}^{h}\left(R_{2}\right)=0
$$

and for $R_{0}<r<R_{1}$,

$$
D_{n} \beta_{n}{ }^{h}\left(R_{1}\right)+A_{n} \beta_{n}{ }^{j}\left(R_{1}\right)-B_{n} \beta_{n}{ }^{h}\left(R_{2}\right)=0
$$

The coefficients $D_{n}$ are determined by the boundary conditions on the surface of the conducting sphere $r=R_{0}$. The excitation source is inside the system (18), (19) is preliminarily resolved with respect to $A_{n}, B_{n}$ :

$$
\begin{aligned}
& A_{n}=D_{n} \sigma_{n}\left(R_{1}, R_{2}\right), \\
& B_{n}=D_{n} \gamma_{n}\left(R_{1}, R_{2}\right),
\end{aligned}
$$

Where: $\sigma_{n}=\frac{\alpha_{n}{ }^{h}\left(R_{2}\right) \beta_{n}{ }^{h}\left(R_{1}\right)-\alpha_{n}{ }^{h}\left(R_{1}\right) \beta_{n}{ }^{h}\left(R_{2}\right)}{\alpha_{n}{ }^{j}\left(R_{1}\right) \beta_{n}{ }^{h}\left(R_{2}\right)-\alpha_{n}{ }^{h}\left(R_{2}\right) \beta_{n}{ }^{j}\left(R_{1}\right)}$,

$$
\gamma_{n}=\frac{\alpha_{n}{ }^{j}\left(R_{1}\right) \beta_{n}{ }^{h}\left(R_{1}\right)-\alpha_{n}{ }^{h}\left(R_{1}\right) \beta_{n}{ }^{j}\left(R_{1}\right)}{\alpha_{n}{ }^{j}\left(R_{1}\right) \beta_{n}{ }^{h}\left(R_{2}\right)-\alpha_{n}{ }^{h}\left(R_{2}\right) \beta_{n}{ }^{j}\left(R_{1}\right)},
$$

Such that, $\sigma_{n}$ can be considered as the reflection coefficient of the nth harmonic from the plasma layer, and $\gamma_{n}$-accordingly, as the harmonic transmission coefficient to the free space through the plasma layer. From $\sigma_{n}$ and $\gamma_{n}$, we can consider $\alpha_{n}$ as absorption coefficient as follows: $\alpha_{n}=1-\sigma_{n}-\gamma_{n}$, By taking into consideration the (4), (5), and (21), the total field inside the dielectric cover can be written as follows:

$$
H_{e}=\sum_{n=1}^{\infty} D_{n}\left\{h_{n}\left(k_{0} \stackrel{r}{r}\right)+\sigma_{n} j_{n}\left(k_{0} \stackrel{r}{r}\right)\right\}\left(R_{0}<r<R_{1}\right)
$$


When $r=R_{0}$, the tangent component of the electric field is related to the voltage across the slot $U$ and the boundary conditions by the relation:

$$
E_{e \theta}=\frac{U}{R_{0}} \delta\left(\theta-\theta_{0}\right)
$$

where: $\delta(x)$ - Dirac's delta function.

The external field in accordance with (6) and (22) can be written as follows:

$$
H_{e}=\sum_{n=1}^{\infty} D_{n} \gamma_{n} h_{n}\left(k_{e} \stackrel{r}{r}\right)\left(R_{2}<r<\infty\right)
$$

To find the radiation pattern, we use the asymptotic behavior of the Bessel functions: $\lim H_{n+\frac{1}{2}}^{(2)}(z)=\sqrt{\frac{2}{\pi z}} e^{-i z} e^{i \pi(n+1) / 2}(z \rightarrow \infty)$

We get for a field in the far zone the following expression

$$
H_{e}=\frac{i e^{-i k_{e} r}}{r} \sum_{n=1}^{\infty} D_{n} \gamma_{n} i^{n} P_{n}^{1}(\cos \theta)
$$

$F(\theta)$ - the radiation pattern of the electric field:

$$
F(\theta)=4 \pi i W \sum_{1}^{\infty} D_{n} \gamma_{n} i^{n} P_{n}^{1}(\cos \theta)
$$

\section{RESULTS AND ANALYSIS}

\subsection{Plasma is switched off}

In this section, the plasma is assumed to be switched off and this means that the spherical slotted antenna is coated by a layer of dielectric material only. The proposed spherical slotted antenna is coated by a dielectric layer at different thickness values to investigate which thickness is suitable to allow the electromagnetic waves to propagate towards the free space with maximum radiation pattern. From Figure 2, the radiation pattern $|F(\theta)|$ is increased and decreased with respect to the increment and decrement in thickness of dielectric layer (Td) in such manner different from what is known. Therefore, it is clear to see that the value of the radiation patter gets increased when decreasing the thickness of the dielectric layer. On the other hand, the radiation pattern gets decreased when increasing the thickness of the dielectric layer. The maximum value of radiation pattern is achieved when the thickness reaches a value of $\lambda / 6$. Therefore the optimum thickness of dielectric layer for such antenna is $\lambda / 6$, i.e. $5 \mathrm{~cm}$.

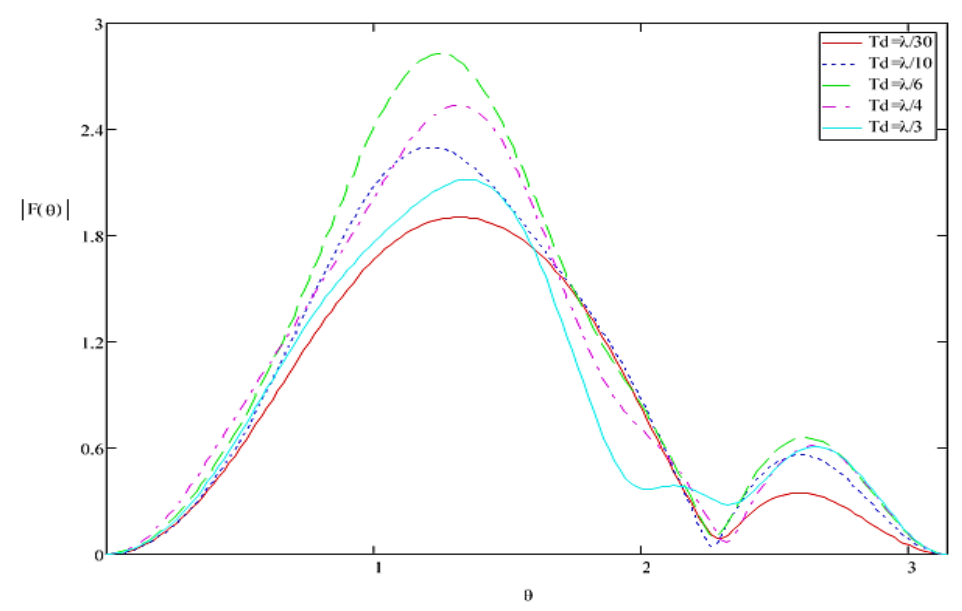

Figure 2. The antenna radiation pattern at different thickness values (Td) of dielectric material layer 


\subsection{Plasma is switched on}

For this section where plasma is switched on, the proposed system consists of spherical slotted antenna coated by layers of dielectric material and plasma. An investigation of the radiation pattern of the proposed system is done under different values of electron plasma density. The authors considered the optimal thickness of dielectric layer $\left(R_{1}\right)$ as $\lambda / 6(5 \mathrm{~cm})$, to become with respect to geometric antenna system as $R_{1}=R_{o}+\lambda / 6$, where this dielectric thickness results in the highest radiation pattern value as shown in Figure 2. Moreover, the plasma layer thickness $\left(R_{2}\right)$ is considered to be $\lambda / 15$ [16] to become with respect to geometric antenna system as $R_{2}=R_{1}+\lambda / 15$.

We can obviously see from Figure 3 that the higher the plasma electron density (ne), the lower the value of the radiation pattern $|\mathrm{F}(\theta)|$ for this proposed antenna system. In case of the electron plasma density (ne) equal to $1010 \mathrm{~m}-3$, there is a good radiation pattern in spite of the appearance of side lobes. From Figure 3, good radiation is obtained when the angular frequency is greater than the angular plasma frequency $(\omega \geq \omega \mathrm{i})$ and this fact is clearly proved in Figure 4 shown when getting good transmission as a result of having small reflection and absorption coefficients.

The accepted reasonable radiation pattern continued until the radiation value reaches zero at $n_{e}=10^{14}$ $\mathrm{m}^{-3}$, where there is not any transmitted electromagnetic waves from antenna system to free space. Moreover, this case takes place when the angular frequency is less than the angular plasma frequency $\left(\omega<\omega_{\mathrm{i}}\right)$. The decrement in the radiation pattern value is directly related to the reflection and absorption caused by the layer of plasma at this plasma concentration. The high density of the plasma layer density caused the difficulty of the electromagnetic waves to pass through this layer due to the slow motion of the electrons in that heavy plasma layer. Consequently, the motion of the plasma electrons become very restricted till it becomes impossible for electromagnetic waves to flow more inside the plasma layer causing zero transmission coefficient $\gamma(\theta)$ and huge reflection and absorption coefficients represented by $\sigma(\theta)$ and $\alpha(\theta)$, respectively, as shown in Figure 5.

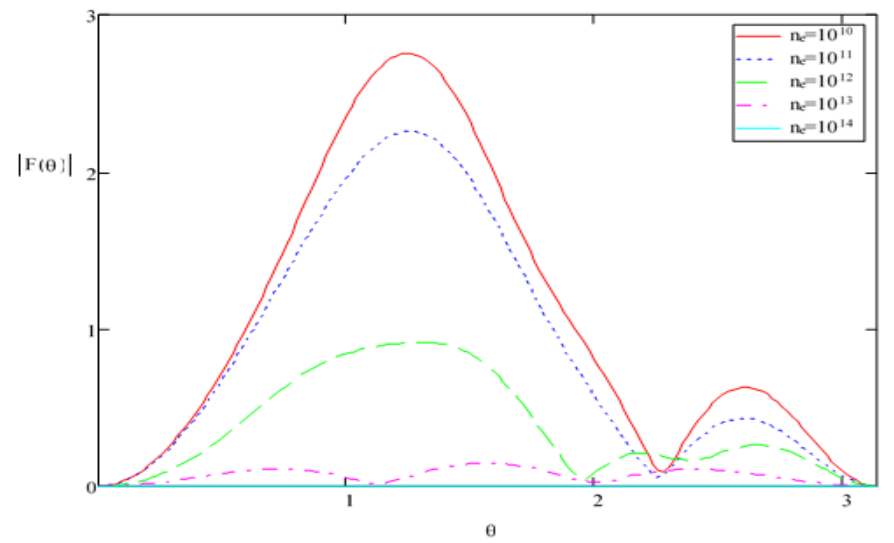

Figure 3. The radiation pattern at different values of plasma concentration (ne)

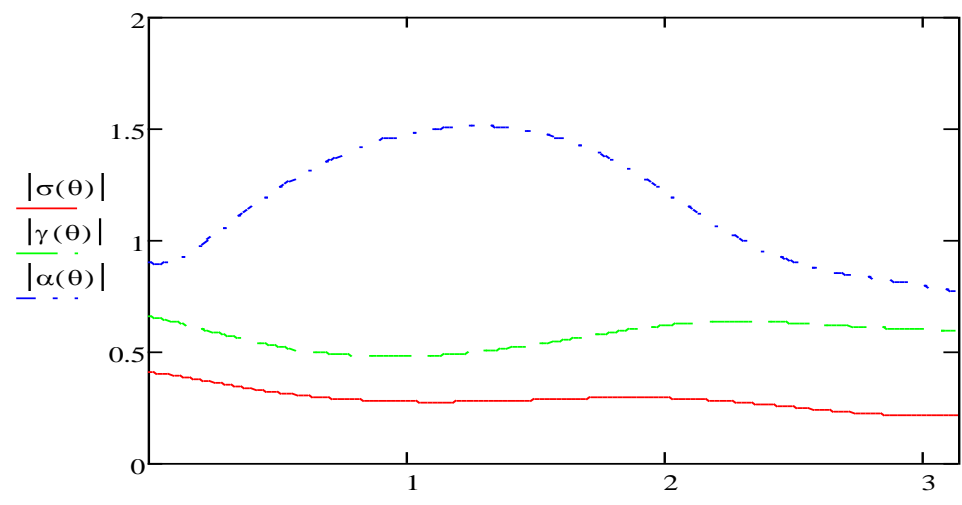

Figure 4. The coefficients of reflection, transmission and absorption at $n_{e}=10^{10} \mathrm{~m}^{-3}$ 


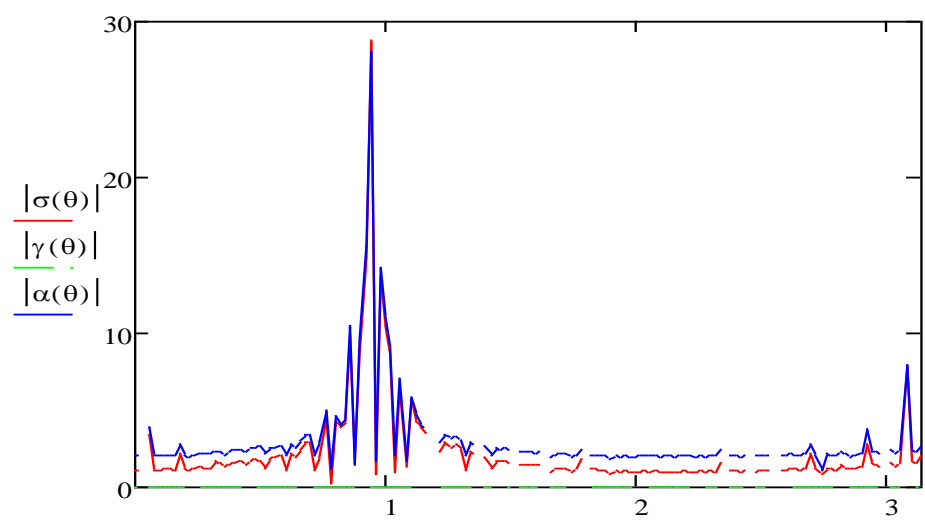

$\theta$

Figure 5. The coefficients of reflection, transmission and absorption at $n_{e}=10^{14} \mathrm{~m}^{-3}$

In order to improve the operation of such antenna for this situation (ne=1014 m-3), allowing the electromagnetic waves to pass to the free space in accepted value of radiation pattern, the authors changed the frequency to become $30 \mathrm{THz}$ instead of $1 \mathrm{GHz}$ and the results are interesting as shown in Figure 6 and Figure 7, where the electromagnetic waves could passes through our antenna system to free space with accepted value of radiation pattern and transmission coefficient $\gamma(\theta)$ when the condition $\omega \geq \omega$ i is met again when increasing the frequency to become in THz. Additionally, the propagated electromagnetic are remarkably continue to spread to dozens of meters through plasma layers almost without slight reduction in the level of radiation as shown in Figure 6.

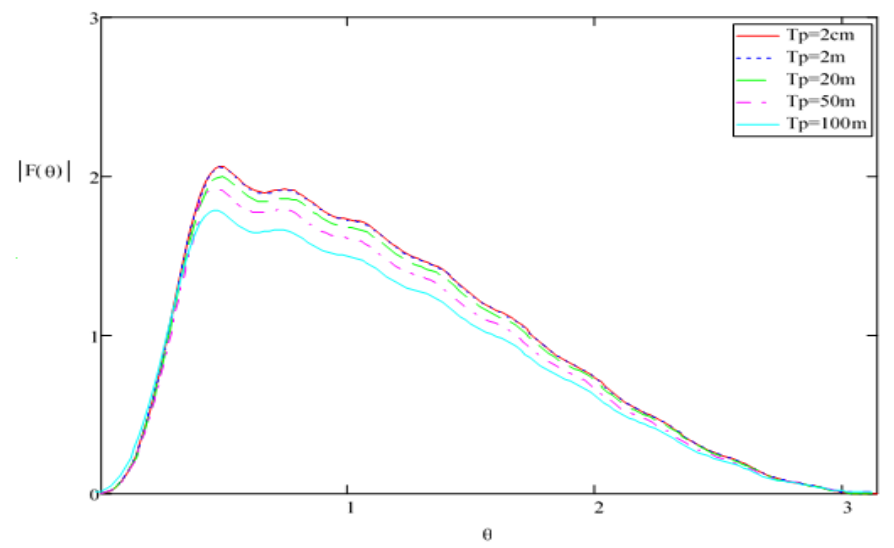

Figure 6. The radiation pattern at different values of thickness of dielectric material layer at $\mathrm{n}_{\mathrm{e}}=10^{14} \mathrm{~m}^{-3}$

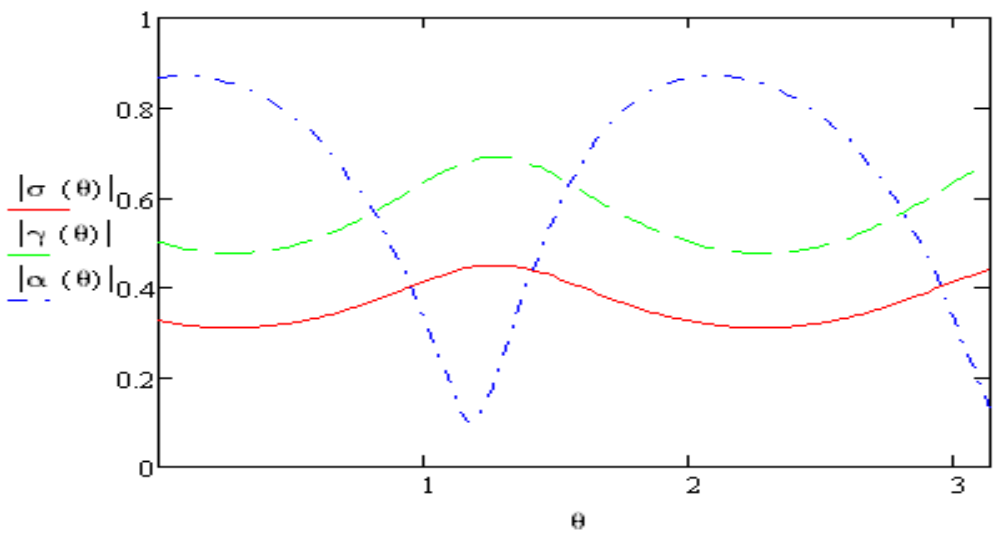

Figure 7. The coefficients of reflection, transmission and absorption at $n_{e}=10^{14}$ 


\section{CONCLUSION}

In conclusion, the proposed new spherical slotted antenna covered by the layers of dielectric material and plasma has reflected great performance operating at a frequency of $1 \mathrm{GHz}$ when the thickness of dielectric layer is not less or more than $\lambda / 6$. However, the proposed antenna system could not maintain this performance when working in special circumstances such as the high density of plasma layer due to the fact that this heavy plasma does not allow the electromagnetic waves to propagate. Thus, the authors suggest using $30 \mathrm{THz}$ operating frequency which is considered as an important additional feature to such antenna due to the wide military applications of this operation frequency.

\section{REFERENCES}

[1] Y. Mushiake and R. E. Webster, "Radiation characteristics with power gain for slots on a sphere," IRE Trans. On antennas and Propagation AP-5, pp.47-55, 1957.

[2] K. W. Leung, "Theory and experiment of a rectangular slot on a sphere," IEEE Trans. Microwave Theory and Tech., vol. 46, pp. 2117-2123, 1998.

[3] Baladel, v."Electromagnetic fields" (McGraw-Hill, 1964), pp. 338-341, 1964.

[4] C. Phongcharoenpanich, M. Krairiksh and J. Takada, "Dyadic Green's functions of a concentric conducting spherical cavity," Proc.1997 Asia- Pacific Microwave Conference, vol. 2, pp. 757-760, Dec. 1997.

[5] Karr, P. R.: "Radiation properties of spherical antennas as a function of the location of the driving force," Res. Nat. Bur. Stand, 46, pp. 422-436, 1951.

[6] Manju Bhaskar, Thomaskutty Mathew," Microstrip multi-stopband filter based on tree fractal slotted resonator," International Journal of Electrical and Computer Engineering, Vol 9, No 5, 2019.

[7] K. W. Leung, "Analysis of the zonal and rectangular slots on a conducting spherical cavity," IEEE Trans. Antennas and Propagate, vol. 49, pp. 1739-1745, 2001.

[8] Ekajit Khoomwong and Chuwong Phongcharoenpanich, "Analysis of an Arbitrarily Rotated Slot on a Conducting Spherical Cavity," 2007.

[9] K. W. Leung, "Rectangular and zonal slots on a sphere with a backing shell: theory and experiment", IEEE Transactions on Antennas and Propagation, 51(7):1434 - 1442, August 2003.

[10] Jalal Naghar, Otman Aghzout, Azzeddin Naghar, "Design Study of a Miniaturized Multi-layered Antenna-inpackage for 2.4 GHZ Wireless Communication," International Journal of Electrical and Computer Engineering, Vol 8, No 5, 2018.

[11] Slavko Rupčić, Vanja Mandrić, Davor Vinko, "Radiation Pattern of Waveguide Antenna Arrays on Spherical Surface - Experimental Results," International Journal of Electrical and Computer Engineering Systems, Vol. 1, No. 1, June 2010.

[12] Ahmed M. Dinar, A. S. Mohd Zain, F. Salehuddin, "Comprehensive identification of sensitive and stable ISFET sensing layer high-k gate based on ISFET/electrolyte models," International Journal of Electrical and Computer Engineering, Vol 9, No 2, 2019.

[13] S. L. Berdnik, V. S. Vasylkovskyi, M. V. Nesterenko and Yu. M. Penkin, "Radiation fields of the spherical slot antenna in a material medium," X International Conference on Antenna Theory and Techniques, Kharkiv, Ukraine, 2015.

[14] N. M. Sahar, M.T. Islam, N. Misran, "Design of dualband antenna for RFID applications," International Journal of Electrical and Computer Engineering, Vol 9, No 4, 2019.

[15] S. J. Towaij, M.Sc, and Prof. M. A. K. Hamid, Ph.D. "Diffraction by a multilayered dielectric-coated sphere with an azimuthal slot," IEEE, Vol.118, No.9, P. 1209-1214, 1971.

[16] O.Sh. Dautov, M. S. Al-abadi, "Directional properties of a slot antenna with a dielectric coating on a metal sphere depending on frequency and coverage parameters," ISSN 2078-6255. Вестник КГТУ им. А.Н. Туполева. о. 4. p.172-177. 2018. (in russian).

[17] J. P. Rybak and R. J. Churchill, "Progress in reentry communications," IEEE Transactions on Aerospace and Electronic Systems, vol. 7, pp. 879-894, 1971.

[18] B. DeWitt and W. Burnside, "Electromagnetic scattering by pyramidal and wedge absorber," IEEE Trans. Antennas Propag., vol. 36, no. 7, pp. 971-984, 1988.

[19] W. W. Salisbury, "Absorbent Body for Electromagnetic Waves," U.S. Patent 2-599-944, 10, 1952.

[20] N. Engheta, "Thin absorbing screens using metamaterial surfaces," in Proc. IEEE Antennas Propag. Soc. Int. Symp., pp. 392-395. 2002.

[21] Edalati, A. and K. Sarabandi, "Wideband, wide angle, polarization independent RCS reduction using nonabsorptive miniaturized-element frequency selective surfaces." IEEE Transactions on Antennas and Propagation, vol. 62, pp. 747-754, 2014.

[22] M. Laroussi, "Interaction of microwaves with atmospheric pressure plasmas," International Journal of Infrared and Millimeter Waves, vol. 16, pp. 2069-2083, 1995.

[23] T. M. Smith, and K. E. Golden, "Radiation Patterns from a Slotted Cylinder Surrounded by a Plasma Sheath," IEEE Transactions on Antennas and Propagation, vol.13, pp. 775-780, Sep. 1965.

[24] Calvin T. Swift, "Radiation Patterns of a Slotted-cylinder Antenna in the Presence of an Inhomogeneous Lossy Plasma," IEEE Transactions on Antennas and Propagation, vol.12, pp. 728-738, Nov. 1964.

[25] John W. Marini, "Radiation and Admittance of an Insulated Slotted-Sphere Antenna Surrounded by a Strongly Ionized Plasma Sheath," National Bureau Of Standards- D. Radio Propagation, No.5, P. 525-532, 1960.

[26] Femila Roseline J, N M. Nandhitha, "Design of Linear Plasma Position Controllers with Intelligent Feedback Systems for Aditya Tokamak," International Journal of Electrical and Computer Engineering, Vol 8, No 5, 2018.

[27] O.Sh. Dautov, M. S. Al-abadi, "optimal conditions for the operation of slot antenna on the metal sphere under a plasma layer," Scientific and technical bulletin of the volga region, pp.55-60, №.2. 2019.

[28] O.Sh. Dautov. "Equivalence of integral and integro-functional equations of electrodynamic problems of diffraction on inhomogeneous bodies" Izv. universities. Radiophysics. - 1991. - T.34. - №8. -C. 936-946.

[29] G.T.Markov, A.F. Chaplin. "Excitation of electromagnetic waves". -M. - L .: "Energy", - 376s., Il, 1967. 